\title{
Gene Transfection for Stem Cell Therapy
}

\author{
Kiheon Baek ${ }^{1}$ C Chengyi Tu ${ }^{1}$ - Janet Zoldan ${ }^{1} \cdot$ Laura J. Suggs $^{1}$
}

Published online: 27 January 2016

(C) Springer International Publishing AG 2016

\begin{abstract}
Genetic engineering of stem cells is a strategy that holds promise for realizing the potential therapeutic benefits of stem cell therapy. Through precise control of the stem cell genome, stem cells can replace or repair damaged tissues as well as serve as a depot for the sustained delivery of therapeutic molecules. Various individual genes, genome editing techniques, and transfection agents have been studied and developed for use in stem cell gene transfection. The goal for this review is to introduce specific genes and editing techniques used in stem cell therapy. Diverse gene transfection agents such as liposomes, polymers, dendrimers, peptides, inorganic nanoparticles, and physical transfection agents are also discussed with particular focus on stem cell considerations.
\end{abstract}

Keywords Stem cell therapy · Stem cell transfection · Gene transfection $\cdot$ Non-viral transfection

This article is part of the Topical Collection on Stem Cells and Nanotechnologies

Laura J. Suggs

suggs@utexas.edu

Kiheon Baek

kbaek@utexas.edu

Chengyi Tu

ctu@utexas.edu

Janet Zoldan

zjanet@utexas.edu

1 Department of Biomedical Engineering, The University of Texas at Austin, 107 West Dean Keeton, Stop C0800, Austin, TX 78712, USA

\section{Introduction}

Stem cell therapy has become one of the fastest growing fields of research in the world following the first isolation of human embryonic stem cells (hESCs) in 1998. Several adult stem cells, progenitor cells, and induced pluripotent stem cells (iPSCs) have since been isolated and characterized with respect to their potential clinical benefit. Due to the unique characteristics of stem cells, namely self-renewal and differentiation potential, stem cell therapy has the potential to treat cardiac diseases, superficial wounds, neurologic diseases, and type I diabetes [1-3]. Transplanted stem cells can rebuild or replace dead tissues and recover existing cells through paracrine effects. However, stem cell therapy has several limitations that must be resolved prior to clinical use. The stem cell differentiation process in most cases is still necessarily heterogeneous, and ensuring uniformity is critical for preventing tumorigenic potential. The activation of an immune response along with an otherwise inhospitable host environment results in a low viability for the majority of transplanted cells [4]. Many research groups have approached these problems through the development of molecular delivery systems, composed of particles and scaffolds, for inserting useful proteins and genes into stem cells.

Through the use of double-stranded DNA that is integrating or not into the host genome as well as double or singlestranded RNA techniques, biological states of stem cells such as differentiation, self-renewal, and growth can be controlled $[5,6]$. Numerous stem cell genetic engineering strategies have been employed to increase cell survival rate, control differentiation, and produce therapeutic factors with exciting results. Despite positive reported outcomes, the genetic manipulation of stem cells faces several problems for ultimate clinical translation, some of which are common across many applications and some of which are unique. Not only are there challenges 
with increasing the efficiency of transfection, controlling targeting, limiting mutagenic potential, and reducing cytotoxicity, but also whatever delivery system is employed must maintain stem cell differentiation status and viability. Research has therefore largely been on the materials and methods for delivering DNA and RNA in order to improve the therapeutic potential of stem cell therapy.

Gene transfection agents are generally categorized as either viral or non-viral. Viral vectors dominate because of high efficiency and long-term maintenance of expression, but concerns including immunogenicity, carcinogenicity, restricted DNA loading capacity, and high cost for mass production impede their commercialization and clinical use [7-9]. In contrast, non-viral vectors avoid the shortcomings of viral vectors, but the efficiency of transfection is compromised. In the case of stem cell transfection, the rate of successful modification ( $40 \%$ by electroporation, $\sim 20-35 \%$ by cationic polymer and liposome, $80 \%$ by efficient viral vectors) is lower than that for differentiated cells [10]. To optimize the transfection efficiency of stem cells, research into making an ideal nonviral vector which has high efficiency and low cytotoxicity is currently underway.

Herein, we discuss several DNA and RNA agents for stem cell therapy and various non-viral methods for stem cell transfection.

\section{Genes for Stem Cell Therapy}

In order to increase the utility of stem cells as therapeutic agents, various genes encoding transcriptional factors for cellular reprogramming, control of differentiation, or the production of therapeutic proteins can be transfected into stem cells. Genes for increasing the survival rate of transplanted stem cell or to control stem cell homing to sites of therapeutic interest have also been studied (Fig. 1).

\section{Genes for Reprogramming and Directing Differentiation of Stem Cells}

Stem cells for therapeutic purposes can include pluripotent stem cells such as iPSCs and embryonic stem cells (ESCs) as well as multipotent stem cells such as mesenchymal stem cells (MSCs) and tissue-specific progenitor cells. The groundbreaking discovery of iPSCs raised the hope for personalized medicine [11]. IPSCs are a particularly attractive therapeutic cell source because they possess the desirable properties of ESCs including unlimited self-renewal and pluripotency while potentially circumventing immune rejection and ethical issues that are roadblocks to clinical translation. The original reprogramming cocktail contained four genes encoding transcriptional factors: Oct3/4, Sox2, c-Myc, and Klf4 [11]. Later studies revealed that some of these factors are not absolutely required. Yamanaka's group showed that reprogramming can be achieved without c-Myc, and the resulting iPSCs were of high quality and minimally tumorigenic [12]. Another study reported that a single gene, Oct4, is sufficient to achieve reprogramming when supplemented with certain small molecules [13]. Generally, the choice of reprogramming genes needs to be carefully considered since the genes not only determine the reprogramming efficiency and the subsequent quality of iPSCs, but also have an effect on the safety of the cells.

While direct injection of adult stem cells like MSCs into circulation or damaged tissue has achieved positive results [14] and, in some therapeutic applications, has reached advanced clinical trials, pluripotent stem cells (ESCs and iPSCs) require appropriate differentiation, specifically to eliminate the risk of tumorigenesis. Generating relevant cell types can increase the specificity and outcome of both adult and pluripotent stem cell-based therapies. To guide differentiation, various transcription factors for inducing cardiogenesis (Gata4, Mef2c, and Tbx5), osteogenesis (bone morphogenetic proteins, bone morphogenetic protein-2 (BMP2) and BMP7), chondrogenesis (SOX-5, SOX-6, SOX-9), and neurogenesis (SOX-1, SOX-2, SOX-3, miR-124, miR-137, miR-184, and MBD1) are used for stem cell transfection to the respective tissue of interest [15-23]. Differentiated stem cells can then be injected to target tissues and replace damaged cells. Yet, since the differentiation process usually requires sequential and temporal expression of specific gene groups (as was shown for iPSCs generation), the clinical applicability of genetic engineering for directing stem cell differentiation remains an open question, unless the role and specificity of additional master key gene switches can be identified (like MyoD for skeletal muscle).

An alternative approach to stem cell differentiation based on overexpression of transcriptional factors is through gene silencing. Toward this aim, microRNAs are prime candidates for genetic engineering considering their role in controlling the expression of multiple transcription factors and in regulating key biological process including cell differentiation. For

Fig. 1 Application of genetically engineered stem cell for cell therapy. The top panel demonstrates transfection of stem cells to silence inhibitors of mineralization. This was accomplished via a CPP complexation strategy with miRNA (adapted from Suh et al. [64•], with permission from Elsevier). The middle panel shows a proposed scheme for cancer therapy using MSCs as carriers. Tumor necrosis factor (TNF)-related apoptosis-inducing ligand (TRAIL) transfected into MSCs by $\mathrm{PEI}_{600^{-}}$ Cyd was injected to C57BL/6 mice for estimating the efficiency of cancer gene therapy (adapted from $\mathrm{Hu}$ et al. [28], with permission from the American Chemical Society). The bottom panel indicates a schematic flow for synthesis of magnetic nanoparticles which have multiple roles in transfection and magnetic resonance imaging (MRI). Catecholfunctionalized polypeptide (CFP) was used for functionalizing iron oxide nanoparticles, and PEI was attached for plasmid condensation (adapted from Park et al. [76•], with permission from Elsevier) 

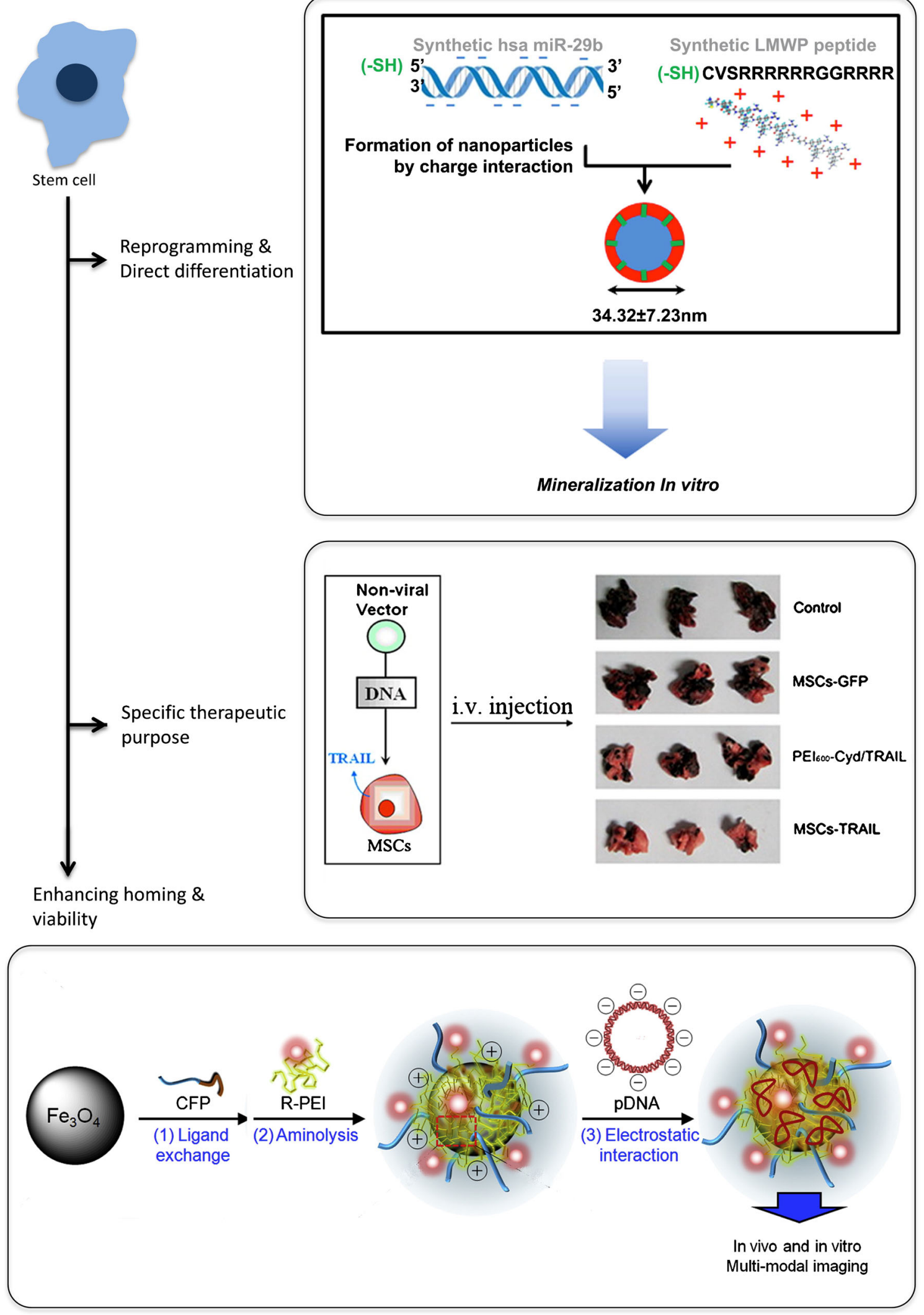

example, ESCs, genetically engineered to overexpress microRNA-1 (a cardiac/muscle-specific miRNA), efficiently differentiated in vivo to cardiomyocytes (100\% more than control ESCs) and contributed to repair of the damaged 
myocardium tissue postmyocardial infarct [24]. We have shown that the heterogeneity of early differentiating hESCs can be significantly reduced through efficient RNA delivery. Specifically, the mesoderm layer formation of embryonic stem cells can be selectively enhanced by as much as 90 -fold through the transfection of siRNA which silences the KDR receptor gene [25].

In the more near term, genetic engineering can greatly benefit stem cell therapy by introducing fluorescent reporter genes under the control of cell type-specific promoters. Such fluorescent cell engineering allows, via fluorescent activated cell sorting, for the identification and enrichment of specific cell populations or the generation of pure cell populations for transplantation by isolating pluripotent stem cells from heterogeneous differentiating cells [26, 27].

\section{Genes for Specific Therapeutic Purposes}

Depending on the purposes of the treatment, therapeutic genes can confer specific properties to stem cells for diverse applications including HIV resistance, angiogenesis, and tumor suppression. Transfected adult stem cells can be particularly well suited for use as a drug delivery vehicle due to their immune privilege [28]. For anti-tumor applications, genetically modified MSCs expressing interleukin-2 (IL-2) augmented the anti-tumor effects of these cells compared to unmodified cells in a mouse glioma model [29]; another study using interferon- $\beta$ (INF- $\beta$ )-expressing MSCs eradicated the tumors in $70 \%$ of treated mice pre-implanted with human ovarian cancer cells [30]. In the area of angiogenic therapy, MSCs transfected by vascular endothelial growth factor (VEGF) plasmid exhibited high angiogenesis potential, increasing vessel densities by 2-4-fold compared to control groups in a mouse hindlimb ischemia model [31]. Likewise, overexpression of fibroblast growth factor-2 (FGF-2) in MSCs significantly improved cell survival by 3 -fold under hypoxic conditions in vitro and expressed cardiac specific markers [32]. More recently, a genetic engineering approach showed promise in treating patients with HIV. IPSCs derived from HIV patients were edited with clustered regularly interspaced short palindromic repeats-Cas9 (CRISPR-Cas9) to derive a mutation in the C-C chemokine receptor type 5 (CCR5) gene, which ultimately granted HIV resistance to the iPSCs and to any cell type differentiated from them [33••].

\section{Genes to Enhance Stem Cell Homing and Viability}

One vital challenge to be resolved for stem cell therapy is the homing of cells to the damaged tissue. The homing capacity of MSCs has been the subject of intense research. Studies have revealed numerous chemokines and growth factors responsible for stem cell homing to damaged myocardium (as reviewed in [34]), including stroma-derived factor- $1 \alpha$ (SDF-
$1 \alpha$ ), chemokine (C-X-C motif) receptor 4 (CXCR4), hepatocyte growth factor (HGF), and FGF-2. Thus, to increase the homing of stem cells, one can genetically overexpress corresponding receptors for these homing signals. These strategies can also be applied to increase homing of ESC- and iPSCderived cells.

In addition to homing, another challenge for cell therapy is that transplanted stem cells frequently have poor survival and incomplete engraftment into injured or diseased tissue because of inflammation, hypoxic stress, and insufficient perfusion. Apoptosis and autophagy may contribute to their low survival rate, but the exact mechanisms initiating these pathways are still unclear. Various proteins, growth factors, and nucleic acids that are related to ischemia, apoptosis, and autophagy have been studied in an attempt to improve stem cell viability. Anti-apoptotic genes such as B cell lymphoma 2 (Bcl-2) and Akt $[35,36]$ and growth factors including VEGF, angiopoietin (Ang-1), and transforming growth factor beta 1 (TGF- $\beta 1$ ) $[37,38]$ have been transfected into stem cells in order to prevent apoptosis and increase survival. In the case of hypoxic stress, methods have been described for overexpressing heme oxygenase (HO-1), which is an anti-oxidant and anti-inflammatory protein, as well as silencing prolyl hydroxylase, a cellular oxygen sensor that controls hypoxia-inducible factor and nuclear factor-kB $[36,39 \bullet]$.

\section{Transfection Methods for Stem Cell Therapy}

The ideal transfection agent must prevent degradation of the delivered gene, penetrate the target cell membrane, and allow for the insertion of the gene into the nucleus. Furthermore, the agent would ideally be harmless to cells [40, 41]. As base materials for non-viral vectors, liposomes, micelles, polymers, dendrimers, peptides, and inorganic nanoparticles have been studied and used for delivery to stem cells. Other techniques such as electroporation, sonoporation, and microfluidic devices for transfection have been developed recently. Gene vectors may traffic via direct penetration into the cell or by endocytosis with the route of entry based on the physical and chemical characteristics of both the cargo and transfection agent. However, the precise relationships between transfection agents and mechanisms are still unclear.

\section{Lipids, Liposomes, and Micelles}

Liposomes, which fulfill several of the criteria for the ideal transfection agent, can be fabricated with various natural and/ or synthetic lipids in order facilitate DNA or RNA encapsulation. Unlike cationic polymers, charged lipids associate via hydrophobic interactions among their aliphatic tails forming liposomes and micelles. Their size $(\sim 100 \mathrm{~nm})$ and the cationic 
character of the head group or modifications thereof facilitate carrying anionic oligonucleotides. Chemical modification techniques such as PEGylation and RGD attachment may limit their clearance from circulation and enhance their targeting ability, respectively [42]. However, since liposomes are usually responsive to environmental conditions such as $\mathrm{pH}$, temperature, salt, and other proteins such as serum, they are unstable in diverse biological systems [9]. While this instability can be used to tune nucleic acid release inside the cell, care must be taken to maintain stability prior to cellular uptake. The positive charge of the majority of cationic liposomes also has a detrimental effect on cell viability; however, surface charge can be altered. Poly(ethyleneimine) (PEI), and metal nanoparticles are commonly used in conjunction with liposomes, the former to avoid cytotoxicity of free PEI and the latter to increase conjugate stability [43, 44]. The advantage of using liposomes as gene vectors is their chemical versatility. Liposomes can be fabricated from a host of different lipids with varying chemistries and tailored to a specific response. Cationic lipids have been designed with hydrophilic head groups such as ammonium groups, polyamines, and guanidinium groups and hydrophobic tail groups including aliphatic chains, steroids, and fluorinated domains. Hydrophilic head groups primarily control the interaction with oligonucleotides and cellular membrane based on their basicity and hydrogen bonding, while hydrophobic tail groups regulate the fluidity and stability of liposomes [9]. These two molecular regions of the lipid influence the liposomes' ultimate transfection efficiency, interaction with oligonucleotides, and cytotoxicity. The versatility of liposome fabrication has led to the development of high-throughput screening techniques. For example, screening of a synthetic lipid library generated by thiol-yne click chemistry revealed that the length of hydrophobic tail (C11 or C12), size of lipoplex (complex of liposome with nucleic acid, between 100 and $200 \mathrm{~nm}$ ), addition of 1,2-dioleoyl-sn-glycero-3-phosphoethanolamine (DOPE), and stable positive charge of lipoplex (above $+50 \mathrm{mV}$ ) all affect the resulting transfection efficiency [45•].

\section{Cationic Polymers and Dendrimers}

Many cationic polymers including poly-L-lysine (PLL) and PEI have been studied for condensing and delivering negatively charged oligonucleotides. The quest to find the ideal gene transfection agent has generated diverse cationic polymers, varying in molecular weight, number of branches, and characteristics of side groups, such as primary, secondary, or tertiary amines. Yet, the high cytotoxicity of these agents caused by positive localized charge has led to the development of several chemical and/or physical modification strategies. These included rendering the polymer backbone biodegradable, generating heterocyclic amine-derivatized polymers and PEGylated polymers [46, 47], crosslinking low-molecularweight polymers with small molecules or lipid grafting [48,
49], and combining cationic polymers with other molecules including lipids [50]. The majority of these modifications are attempted to improve the toxicity of the polymers by making them degradable or rendering the relative size of the agent larger. Cationic polysaccharides have inherent characteristics that are well suited for use as a transfection agent. In general, they are biocompatible, biodegradable, and non-toxic. Some cationic polysaccharides have built in specific cell targeting capabilities. For example, pullulan and curdlan have binding sites for Dectin-1 receptors uniquely found on dendritic cells, macrophages, and B cells [38, 51, 52]. Alternatively, researchers have generated libraries of synthetic biodegradable cationic polymers such as poly beta-amino esters (PBAE) and utilized high-throughput screening strategies to identify candidates that will efficiently transfect ESCs and MSCs [21, 53, $54,55 \bullet \bullet$. A major research thrust involving cationic polymers has been devoted to enhancing the escape of polymer/ oligonucleotide polyplexes from the endosome, one of the leading hurdles for gene transfection. Toward this aim, environmentally sensitive polymers have been designed with improved transfection efficiency and cytotoxicity. During endocytosis, the $\mathrm{pH}$ of the endosome is reduced; such a change can trigger a proton sponge effect in these polymers, which then disrupts the endosomal membrane and leads to release of gene cargo into the cytoplasm. Various amine-modified PEIs having $\mathrm{pKa}$ values from 5 to 6 can cause endosomal escape via osmotic burst [46]. Poly ethylacrylic acids (PEAA) which exhibit a transition in their hydrophilicity at $\mathrm{pH}$ 5-6 can also disrupt the endosomal membrane $[47,56]$.

Dendrimers are large, monodisperse, highly branched molecules. They usually have a globular structure and can exhibit specific functional groups on their surface. Since dendrimers are synthesized through recurrent chemical reactions, their size is predictable, typically 1 to $10 \mathrm{~nm}$ [57]. These strengths make dendrimers advantageous for use as designing gene delivery agents, and many amine group-containing dendrimers such as poly(amidoamine) (PAMAM), melamine-based dendrimers, and polypropylenimine (PPI) dendrimers have been studied [9, 58]. Notably, PAMAM dendrimers have been shown to efficiently deliver interfering RNA to ESCs compared to PEI-based lipopolymer controls. In order to enhance their transfection efficiency and specificity and reduce cytotoxicity, modifications such as PEGylation, RGD attachment, and charge modification have been applied [59, 60].

\section{Peptides}

Since peptides are the molecular building blocks of proteins conferring numerous biological functions, peptides have been investigated as gene transfection agents. In order to interact with oligonucleotides, penetrate the cell membrane, and traffic genes into the nucleus, specific peptide regions are employed. Peptides can be designed to contain DNA-binding domains, 
cell-penetrating peptides (CPPs), also known as peptide transduction domains, and nuclear localization signal (NLS) peptides. CPPs usually have basic amino acids that possess both positive charge and amphipathic secondary structure for interacting with cell membranes and destabilizing them [61]. Various natural and synthetic CPPs including TAT, penetratin, R8 and R9, Pep family, transportan, and GALA have been studied for their ability to transfect genes, used to generate novel synthetic CPPs, and modified through lipid and peptide addition [18, 62, 63, 64•]. However, CPPs with high positive charge can also be cytotoxic and demonstrate non-specific interaction with serum proteins. Recently, novel hydrophobic CPPs from signal peptides and Bax-inhibiting peptides were found. In particular, Bax-inhibiting peptides have been conjugated to non-toxic nanoparticle carriers to improve cellular uptake of gene cargo into MSCs. This is of particular interest as MSCs typically exhibit low transfection efficiencies [10]. The reported role of NLS peptides has been to import proteins into the nucleus, another critical step in the transfection process. These amino acid sequences are also non-toxic and can penetrate cell as well as nuclear membranes, thereby increasing transfection efficiency [65]. Studies are ongoing to determine the precise biophysical mechanisms for peptide insertion into membranes [18, 66, 67].

\section{Inorganic Nanoparticles}

Inorganic solid particles with a 10-1000-nm size have many advantages as gene transfection agents. As a result of a solid core, they are more stable than liposomes and can present a high density of surface ligand. Their small size facilitates cell penetration, and inorganic nanoparticles can protect nucleic acids from degradation during cellular trafficking [68•]. A range of inorganic materials such as gold, silver, calcium phosphate, iron oxide, silica particles, quantum dots, and carbon nanotubes have all been studied as potential gene vectors. The non-toxic and readily modifiable characteristics of gold make them a leading candidate for gene transfection $[10,44]$. Silver, which is known for its bactericidal effect yet can also be cytotoxic to mammalian cells, has been recently used for generating a photo-activated delivery agent. Silver has the advantage of having an approximately ten times greater localized surface plasmon field than gold $[68 \bullet, 69]$. Calcium phosphate, a non-toxic mineral, can interact with DNA and destabilize the endosomal membrane in response to acidic $\mathrm{pH}$. It has been used as transfection agent for over 30 years. Recently, a wide variety of calcium phosphate nanoparticles and scaffolds have been studied for the dual role of increasing transfection efficiency as well as serving as a substrate for tissue regeneration [70•, 71-73]. Iron oxide, another mineral in the form of nanoparticles, has been applied to make multifunctional transfection agents because of its superparamagnetic character. Transfection agents that can be detected through magnetic resonance imaging (MRI) were recently synthesized, and it was demonstrated that applying oscillating magnetic fields improves the transfection efficiency of this agent $[74,75,76 \bullet$. Lastly, the prospect of mesoporous silica particles (MSNs) as effective transfection agents has been studied. The nanometer size of pore channels can contain cargo effectively, and the easily controllable pore size and surface chemistry make MSNs an attractive transfection agent. By using aminated MSNs, Kim et al. showed production of BMP2 in mesenchymal stem cells (MSCs) of $66 \%$ efficiency [15].

\section{Electroporation, Sonoporation, and Microfluidic Devices}

In an effort to develop transfection methods without cytotoxic carriers, several researchers have studied transfection with physical methods such as electroporation, sonoporation, and laser irradiation. Among them, reports on electroporation are most common; however, the high voltages used can cause cell death [77]. Instead, sonoporation and various microfluidic devices have been developed to improve physical gene transfection. In the case of sonoporation, several studies have reported that ultrasound-mediated microbubble burst increases gene transfection efficiency both in vitro and in vivo [78, 79]. Microfabricated fluidic devices have also been used in order to overcome limitations with gene transfection. Sharei et al. used a microfluidic platform that minimally deforms cells in order to make the membrane permeable when cells flow through a narrow path. This platform was then used to introduce reprogramming proteins into fibroblasts, exhibiting a 10-fold improvement in iPSC generation compared to other non-viral methods [80••]. Others have focused on constructing electroporation-based microfluidic devices that reduce the voltage necessary for transfection. $\mathrm{Xi}$ et al. generated a nanoelectroporation platform composed of alumina nanostraws for reducing electroporation voltage and increasing uniformity over a large area [81]. Kang et al. fabricated a two-level electroporation device composed of a cell culture chamber situated on top of a series of microchannels. The microchannels were designated for loading transfection materials and reducing the voltage through localized electroporation using a nanofountain probe. This microfluidic device showed $50 \%$ plasmid transfection efficiency with significantly lower voltage $(10 \mathrm{~V})$ than traditional electroporation [77]. Lastly, Grigsby et al. designed a microfluidic system for producing polymer-nucleic acid nanoparticle that is smaller, more uniform, with less aggregation than otherwise possible. The physical characteristics of nanocomplexes enhanced the transfection efficiency in various cell lines including both stem cells and primary cells [82]. 


\section{Conclusions and Future Directions}

Diverse genes and viral and non-viral methods have been studied for stem cell transfection. As a whole, stem cells are generally more difficult to transfect than their primary cell counterparts and care must be taken to control differentiation status, viability, and tumorigenicity. The focus of gene transfection for stem cell therapy has been the insertion of genes controlling differentiation, preventing apoptosis, enhancing angiogenesis, and encoding therapeutic proteins. Stem cells are transfected in order to increase their survival rate and to subsequently improve therapeutic efficacy.

Future advances will likely involve specific nucleases such as zinc finger nucleases (ZFNs), transcription activator-like effector nucleases (TALENs), and CRISPR/Cas in order to improve the precision of genome editing, prevent mutations and maintain stable, long-term gene expression. Since stem cell mutations, particularly with embryonic stem cells, caused by improper genome editing produces tumors, accurate genome engineering is absolutely essential. In the past decade, engineered nucleases consisting of non-specific nucleases and sequence specific DNA-binding domain have been studied. Among several systems, ZFNs and TALENs that embody a flexible editing system dominate genome engineering. The 2010 discovery of the clustered regularly interspaced short palindromic repeat (CRISPR)/Cas system, which is a bacterial immune mechanism for removing foreign genes, offered a path toward very precise and flexible genome engineering. A system composed of CRISPR/Cas was generated and applied in 2013 [83] Most recently, the Zhang group has identified a new CRISPER enzyme, cfl, that offers a more precise and simpler gene editing than Cas [84]. It will be interesting to see its impact on genetically engineered stem cells. New gene engineering tools such as ZFNs, TALENs, and CRISPR modules offer great potential, yet they still need to be delivered into cells and therefore have been used with various viral and non-viral vectors with similar limitations related to transfection efficiencies [85, 86, 87••, 88-92].

As has been shown previously, it is possible to combine strategies for gene transfection along with other therapeutic purposes such as cell tracking (as in the case of metal nanoparticles), cell sorting (using genes encoding fluorescent proteins), and, more recently, gene delivery via scaffolds that may also allow for cell growth and tissue remodeling. Many biocompatible and functionalized scaffolds, made by electrospinning, lithography, microfabrication, and self-assembly, have been developed for tissue regeneration. Various biocompatible and biodegradable compounds such as poly(lactic-co-glycolic acid) (PLGA), poly-caprolactone (PCL), poly(amido amine), PEG, collagen, and chitosan have been used and studied [17, 93-100]. Researchers have devised gene-activated scaffolds for combination gene and cell therapy. Several characteristics of scaffolds may be particularly well suited toward use in combination with gene therapy including transfection agent preservation, sustained agent presentation via controlled release, and the enhancement of transfection through the maintenance of the proper 3D stem cell microenvironment. Gene-activated biomaterials for stem cell differentiation toward osteogenesis [17, 98, 99] and chondrogenesis $[95,97]$ have been intensively studied due to the requirement of a biomimetic platform. Murphy's group has described a high-throughput 3D scaffold screening system for analyzing diverse transfection factors and optimizing mineral coating for osteogenesis of hMSC [93, 100]. This work may serve to inform future combination strategies.

Various materials containing lipids, polymers, dendrimers, peptides, nanoparticles, and physical transfection agents have been considered as transfection carriers. Of non-viral gene carriers, no one solution has emerged as optimal, and therefore, there is a need for investigation into multifunctional carriers that combine several strategies together. We speculate that such multifunctional carriers can eventually lead to designing transfection agents that are both non-toxic with high transfection efficiency. Considering the unique factors that prevent safe and efficient transfection of stem cells, several research groups have approached the problem by constructing material libraries and combine existing materials in order to have a large search space for obtaining the optimal solution. A large body of existing literature in adult stem cell transfection will serve as the base for understanding gene transfection mechanisms in stem cell transfection.

\section{Compliance with Ethical Standards}

Conflict of Interest Kiheon Baek, Chengyi Tu, Janet Zoldan, and Laura J. Suggs declare that they have no conflict of interest.

Human and Animal Rights and Informed Consent This article does not contain any studies with human or animal subjects performed by any of the authors.

\section{References}

Papers of particular interest, published recently, have been highlighted as:

- Of importance

•. Of major importance

1. Bhasin A, Srivastava MVP, Mohanty S, et al. Stem cell therapy: a clinical trial of stroke. Clin Neurol Neurosurg. 2013;115(7):10038.

2. Chhabra P, Brayman KL. Stem cell therapy to cure type 1 diabetes: from hype to hope. Stem Cells Transl Med. 2013;2(5):328-36.

3. Garbern JC, Lee RT. Cardiac stem cell therapy and the promise of heart regeneration. Cell Stem Cell. 2013;12(6):689-98. 
4. Diekman BO, Guilak F. Stem cell-based therapies for osteoarthritis: challenges and opportunities. Curr Opin Rheumatol. 2013;25(1):119.

5. Robinton DA, Daley GQ. The promise of induced pluripotent stem cells in research and therapy. Nature. 2012;481(7381):295305

6. Mundra V, Gerling IC, Mahato RI. Mesenchymal stem cell-based therapy. Mol Pharm. 2013;10(1):77-89.

7. Bakhshandeh B, Soleimani M, Hafizi M, et al. A comparative study on nonviral genetic modifications in cord blood and bone marrow mesenchymal stem cells. Cytotechnology. 2012;64(5): 523-40.

8. Mellott AJ, Forrest ML, Detamore MS. Physical non-viral gene delivery methods for tissue engineering. Ann Biomed Eng. 2013;41(3):446-68.

9. Ziraksaz Z, Nomani A, Soleimani M, et al. Evaluation of cationic dendrimer and lipid as transfection reagents of short RNAs for stem cell modification. Int J Pharm. 2013;448(1):231-8.

10. Muroski ME, Morgan Jr TJ, Levenson CW, et al. A gold nanoparticle pentapeptide: gene fusion to induce therapeutic gene expression in mesenchymal stem cells. J Am Chem Soc. 2014;136(42): 14763-71.

11. Takahashi K, Yamanaka S. Induction of pluripotent stem cells from mouse embryonic and adult fibroblast cultures by defined factors. Cell. 2006;126(4):663-76.

12. Nakagawa M, Koyanagi M, Tanabe K, et al. Generation of induced pluripotent stem cells without Myc from mouse and human fibroblasts. Nat Biotechnol. 2008;26(1):101-6.

13. Li Y, Zhang Q, Yin X, et al. Generation of iPSCs from mouse fibroblasts with a single gene, Oct4, and small molecules. Cell Res. 2011;21(1):196-204

14. Ankrum J, Karp JM. Mesenchymal stem cell therapy: two steps forward, one step back. Trends Mol Med. 2010;16(5):203-9.

15. Kim T-H, Kim M, Eltohamy M, et al. Efficacy of mesoporous silica nanoparticles in delivering BMP-2 plasmid DNA for in vitro osteogenic stimulation of mesenchymal stem cells. J Biomed Mater Res A. 2013;101(6):1651-60.

16. Li Y, Fan L, Liu S, et al. The promotion of bone regeneration through positive regulation of angiogenic-osteogenic coupling using microRNA-26a. Biomaterials. 2013;34(21):5048-58.

17. Yang X, Han G, Pang X, et al. Chitosan/collagen scaffold containing bone morphogenetic protein-7 DNA supports dental pulp stem cell differentiation in vitro and in vivo. J Biomed Mater Res A, 2012.

18. Guo X, Chu X, Li W, et al. Chondrogenic effect of precartilaginous stem cells following NLS-TAT cell penetrating peptide-assisted transfection of eukaryotic hTGFbeta3. J Cell Biochem. 2013;114(11):2588-94.

19. Scheibe F, Gladow N, Mergenthaler P, et al. Nonviral gene delivery of erythropoietin by mesenchymal stromal cells. Gene Ther. 2012;19(5):550-60.

20. Li L, Krymskaya L, Wang J, et al. Genomic editing of the HIV-1 coreceptor CCR5 in adult hematopoietic stem and progenitor cells using zinc finger nucleases. Mol Ther. 2013;21(6):1259-69.

21. Tzeng SY, Hung BP, Grayson WL, et al. Cystamine-terminated poly(beta-amino ester)s for siRNA delivery to human mesenchymal stem cells and enhancement of osteogenic differentiation. Biomaterials. 2012;33(32):8142-51.

22. Sonoyama T, Sone M, Honda K, et al. Differentiation of human embryonic stem cells and human induced pluripotent stem cells into steroid-producing cells. Endocrinology. 2012;153(9):433645.

23. Yang Y-P, Chien Y, Chiou G-Y, et al. Inhibition of cancer stem cell-like properties and reduced chemoradioresistance of glioblastoma using microRNA145 with cationic polyurethane-short branch PEI. Biomaterials. 2012;33(5):1462-76.
24. Glass C, Singla DK. MicroRNA-1 transfected embryonic stem cells enhance cardiac myocyte differentiation and inhibit apoptosis by modulating the PTEN/Akt pathway in the infarcted heart. Am J Physiol Heart Circ Physiol. 2011;301(5):H2038-49.

25. Zoldan J, Lytton-Jean AK, Karagiannis ED, et al. Directing human embryonic stem cell differentiation by non-viral delivery of siRNA in 3D culture. Biomaterials. 2011;32(31):7793-800.

26. Jha R, Wile B, Wu Q, et al. Molecular beacon-based detection and isolation of working-type cardiomyocytes derived from human pluripotent stem cells. Biomaterials. 2015;50:176-85.

27. Wu TJ, Tzeng YK, Chang WW, et al. Tracking the engraftment and regenerative capabilities of transplanted lung stem cells using fluorescent nanodiamonds. Nat Nanotechnol. 2013;8(9):682-9.

28. Hu Y-L, Huang B, Zhang T-Y, et al. Mesenchymal stem cells as a novel carrier for targeted delivery of gene in cancer therapy based on nonviral transfection. Mol Pharm. 2012;9(9):2698-709.

29. Nakamura K, Ito Y, Kawano Y, et al. Antitumor effect of genetically engineered mesenchymal stem cells in a rat glioma model. Gene Ther. 2004;11(14):1155-64.

30. Dembinski JL, Wilson SM, Spaeth EL, et al. Tumor stroma engraftment of gene-modified mesenchymal stem cells as anti-tumor therapy against ovarian cancer. Cytotherapy. 2013;15(1):20-32.

31. Yang F, Cho SW, Son SM, et al. Genetic engineering of human stem cells for enhanced angiogenesis using biodegradable polymeric nanoparticles. Proc Natl Acad Sci U S A. 2010;107(8): 3317-22.

32. Song H, Kwon K, Lim S, et al. Transfection of mesenchymal stem cells with the FGF-2 gene improves their survival under hypoxic conditions. Mol Cells. 2005;19(3):402-7.

33.• Ye L, Wang J, Beyer AI, et al. Seamless modification of wild-type induced pluripotent stem cells to the natural CCR5Delta32 mutation confers resistance to HIV infection. Proc Natl Acad Sci U S A. 2014;111(26):9591-6. In this paper, engineered iPSCs which are resistant to $\mathrm{HIV}-1$ infection were produced by using piggyBac technology with TALENs and CRISPR-Cas9 system. Genome editing was successfully progressed without detectable exogenous sequences and high efficiency, and this paper showed a promising autologous therapy in HIV infection.

34. Penn MS, Mangi AA. Genetic enhancement of stem cell engraftment, survival, and efficacy. Circ Res. 2008;102(12):1471-82.

35. Fang Z, Yang Q, Luo W, et al. Differentiation of GFP-Bcl-2engineered mesenchymal stem cells towards a nucleus pulposuslike phenotype under hypoxia in vitro. Biochem Biophys Res Commun. 2013;432(3):444-50.

36. Samper E, Diez-Juan A, Montero JA, et al. Cardiac cell therapy: boosting mesenchymal stem cells effects. Stem Cell Rev. 2013;9(3):266-80.

37. Alaiti MA, Ishikawa M, Masuda $\mathrm{H}$, et al. Up-regulation of miR210 by vascular endothelial growth factor in ex vivo expanded CD34+ cells enhances cell-mediated angiogenesis. J Cell Mol Med. 2012;16(10):2413-21.

38. Deng WW, Cao X, Wang M, et al. Delivery of a transforming growth factor beta-1 plasmid to mesenchymal stem cells via cationized Pleurotus eryngii polysaccharide nanoparticles. Int J Nanomedicine. 2012;7:1297-311.

39. Wang WE, Yang D, Li L, et al. Prolyl hydroxylase domain protein 2 silencing enhances the survival and paracrine function of transplanted adipose-derived stem cells in infarcted myocardium. Circ Res. 2013;113(3):288-300. In this work, prolyl hydroxylase domain protein 2 (PHD2) was studied for promoting adipose derived stem cells survival in infarcted hearts and increasing their paracrine function. This research showed that PHD2 silencing could be a new strategy to improve stem cell therapy for cardiac repair after myocardial infarction.

40. Peng L-H, Tsang S-Y, Tabata Y, et al. Genetically-manipulated adult stem cells as therapeutic agents and gene delivery vehicle 
for wound repair and regeneration. J Control Release. 2012;157(3):321-30.

41. Malmsten M. Inorganic nanomaterials as delivery systems for proteins, peptides, DNA, and siRNA. Curr Opin Colloid Interface Sci. 2013;18(5):468-80.

42. Dash TK, Konkimalla VB. Nanoformulations for delivery of biomolecules: focus on liposomal variants for siRNA delivery. Critical Reviews ${ }^{\mathrm{TM}}$ in Therapeutic Drug Carrier Systems 2013; $30(6)$.

43. Elsler S, Schetting S, Schmitt G, et al. Effective, safe nonviral gene transfer to preserve the chondrogenic differentiation potential of human mesenchymal stem cells. J Gene Med. 2012;14(7):501-11.

44. Vankayala R, Chiang C-S, Chao J-I, et al. A general strategy to achieve ultra-high gene transfection efficiency using lipidnanoparticle composites. Biomaterials. 2014;35(28):8261-72.

45. Li L, Zahner D, Su Y, et al. A biomimetic lipid library for gene delivery through thiol-yne click chemistry. Biomaterials. 2012;33(32):8160-6. In this paper, a rapid and cost effective way of synthesis and screening lipid library for stem cell transfection was produced. Hundreds of lipids and specific characteristics for increasing transfection efficiency were estimated.

46. Salmasi Z, Shier WT, Hashemi M, et al, Heterocyclic aminemodified polyethylenimine as gene carriers for transfection of mammalian cells. Eur J Pharm Biopharm 2015.

47. Yue $\mathrm{Y}, \mathrm{Wu} \mathrm{C}$. Progress and perspectives in developing polymeric vectors for in vitro gene delivery. Biomater Sci. 2013;1(2):15270.

48. Hu J, Kovtun A, Tomaszewski A, et al. A new tool for the transfection of corneal endothelial cells: calcium phosphate nanoparticles. Acta Biomater. 2012;8(3):1156-63.

49. KC Remant B, Kucharski C, Uludağ H. Additive nanocomplexes of cationic lipopolymers for improved non-viral gene delivery to mesenchymal stem cells. J Mater Chem B. 2015;3(19):3972-82.

50. Song H, Wang G, He B, et al. Cationic lipid-coated PEI/DNA polyplexes with improved efficiency and reduced cytotoxicity for gene delivery into mesenchymal stem cells. Int J Nanomedicine. 2012;7:4637-48.

51. Zhang T-Y, Huang B, Yuan Z-Y, et al. Gene recombinant bone marrow mesenchymal stem cells as a tumor-targeted suicide gene delivery vehicle in pulmonary metastasis therapy using non-viral transfection. Nanomedicine. 2014;10(1):257-67.

52. Han J, Cai J, Borjihan W, et al. Preparation of novel curdlan nanoparticles for intracellular siRNA delivery. Carbohydr Polym. 2015;117:324-30.

53. Keeney M, Onyiah S, Zhang Z, et al. Modulating polymer chemistry to enhance non-viral gene delivery inside hydrogels with tunable matrix stiffness. Biomaterials. 2013;34(37):9657-65.

54. Keeney M, Deveza L, Yang F. Programming stem cells for therapeutic angiogenesis using biodegradable polymeric nanoparticles. J Vis Exp. 2013;79:e50736.

55.• Park H-J, Yang F, Cho S-W. Nonviral delivery of genetic medicine for therapeutic angiogenesis. Adv Drug Deliv Rev. 2012;64(1): 40-52. In this work, combinatorial libraries of PBAEs were constructed for screening an effective non-viral transfection agent and tested in various cell lines. Additionally, in vivo cell therapy with engineered hMSCs in a mouse model demonstrated remarkable results.

56. Forbes DC, Peppas NA. Polycationic nanoparticles for siRNA delivery: comparing ARGET ATRP and UV-initiated formulations. ACS Nano. 2014;8(3):2908-17.

57. Lehner R, Wang X, Marsch S, et al. Intelligent nanomaterials for medicine: carrier platforms and targeting strategies in the context of clinical application. Nanomedicine. 2013;9(6):742-57.

58. Akhtar S, Chandrasekhar B, Attur S, et al. On the nanotoxicity of PAMAM dendrimers: Superfect(R) stimulates the EGFR-ERK1/2 signal transduction pathway via an oxidative stress-dependent mechanism in HEK 293 cells. Int J Pharm. 2013;448(1):239-46.

59. Biswas S, Deshpande PP, Navarro G, et al. Lipid modified triblock PAMAM-based nanocarriers for siRNA drug co-delivery. Biomaterials. 2013;34(4):1289-301.

60. Kong L, Alves CS, Hou W, et al. RGD peptide-modified dendrimer-entrapped gold nanoparticles enable highly efficient and specific gene delivery to stem cells. ACS Appl Mater Interfaces. 2015;7(8):4833-43.

61. Gabrielson NP, Lu H, Yin L, et al. Reactive and bioactive cationic alpha-helical polypeptide template for nonviral gene delivery. Angew Chem Int Ed Engl. 2012;51(5):1143-7.

62. Yamano S, Dai J, Hanatani S, et al. Long-term efficient gene delivery using polyethylenimine with modified Tat peptide. Biomaterials. 2014;35(5):1705-15.

63. Veiman K-L, Mager I, Ezzat K, et al. PepFect14 peptide vector for efficient gene delivery in cell cultures. Mol Pharm. 2013;10(1): 199-210.

64. Suh JS, Lee JY, Choi YS, et al. Peptide-mediated intracellular delivery of miRNA-29b for osteogenic stem cell differentiation. Biomaterials. 2013;34(17):4347-59. In this paper, a low molecular weight protamine (LMWP) transfection system was produced for overcoming limitations with cell penetrating peptides. LMWP/miR-29b showed efficient delivery into hMSCs and osteoblastic differentiation in vitro.

65. Lo W-L, Chien Y, Chiou G-Y, et al. Nuclear localization signalenhanced RNA interference of EZH2 and Oct4 in the eradication of head and neck squamous cell carcinoma-derived cancer stem cells. Biomaterials. 2012;33(14):3693-709.

66. Yin J, Meng X, Zhang S, et al. The effect of a nuclear localization sequence on transfection efficacy of genes delivered by cobalt(II)polybenzimidazole complexes. Biomaterials. 2012;33(31):7884 94.

67. Zhang H, Liang Z, Li W, et al. Nuclear location signal peptidemodified poly (ethyleneimine)/DNA complexes: an efficient gene delivery vector in vitro and in vivo. J Bioact Compat Polym. 2013;28(3):218-32.

68. Qureshi AT, Monroe WT, Dasa V, et al. miR-148b-nanoparticle conjugates for light mediated osteogenesis of human adipose stromal/stem cells. Biomaterials. 2013;34(31):7799-810. In this work, photo-activated microRNA silver nanoparticle delivery system was developed and tested for stem cell differentiation into an osteogenic linage. This system showed not only high transfection efficiency and biocompatibility but also elaborate control with light activation. Various methods such as histological staining, Q-PCR, confocal microscope were used for confirming light triggering transfection system.

69. Qureshi AT, Doyle A, Chen C, et al. Photoactivated miR-148bnanoparticle conjugates improve closure of critical size mouse calvarial defects. Acta Biomater. 2015;12:166-73.

70. Cao X, Deng W, Qu R, et al. Non-viral co-delivery of the four Yamanaka factors for generation of human induced pluripotent stem cells via calcium phosphate nanocomposite particles. Adv Funct Mater. 2013;23(43):5403-11. In this work, a simple, safe, and efficient calcium phosphate nanoparticle transfection system was produced for reprogramming the stem cells. Conclusively, a higher reprogramming efficiency $(0.049 \%)$ and successful in vitro and in vivo experiments were shown.

71. Cao X, Deng W, Wei Y, et al. Incorporating pTGF- $\beta 1 /$ calcium phosphate nanoparticles with fibronectin into 3-dimensional collagen/chitosan scaffolds: efficient, sustained gene delivery to stem cells for chondrogenic differentiation. Eur Cells Mater. 2012;23:81-93.

72. Wang $\mathrm{X}$, Ito $\mathrm{A}, \mathrm{Li} \mathrm{X}$, et al. DNA-lipid-apatite composite layers enhance gene expression of mesenchymal stem cells. Mater Sci Eng C. 2013;33(1):512-8. 
73. Curtin CM, Cunniffe GM, Lyons FG, et al. Innovative collagen nano-hydroxyapatite scaffolds offer a highly efficient non-viral gene delivery platform for stem cell-mediated bone formation. Adv Mater. 2012;24(6):749-54.

74. Yiu HHP, Pickard MR, Olariu CI, et al. Fe3O4-PEI-RITC magnetic nanoparticles with imaging and gene transfer capability: development of a tool for neural cell transplantation therapies. Pharm Res. 2012;29(5):1328-43.

75. Adams CF, Pickard MR, Chari DM. Magnetic nanoparticle mediated transfection of neural stem cell suspension cultures is enhanced by applied oscillating magnetic fields. Nanomedicine. 2013;9(6):737-41

76. Park W, Yang HN, Ling D, et al. Multi-modal transfection agent based on monodisperse magnetic nanoparticles for stem cell gene delivery and tracking. Biomaterials. 2014;35(25):7239-47. In this paper, improved transfection efficiency and biocompatibility of a multi-modal transfection agent (MTA) was developed. In vivo experiments showed successful MTA-hMSC visualization after transplantation over 14 days.

77. Kang W, Giraldo-Vela JP, Nathamgari SSP, et al. Microfluidic device for stem cell differentiation and localized electroporation of postmitotic neurons. Lab Chip. 2014;14(23):4486-95.

78. Li P, Gao Y, Liu Z, et al. DNA transfection of bone marrow stromal cells using microbubble-mediated ultrasound and polyethylenimine: an in vitro study. Cell Biochem Biophys. 2013;66(3):775-86.

79. Shapiro G, Kallai I, Sheyn D, et al. Ultrasound-mediated transgene expression in endogenous stem cells recruited to bone injury sites. Polym Adv Technol. 2014;25(5):525-31.

80.• Sharei A, Zoldan J, Adamo A, et al. A vector-free microfluidic platform for intracellular delivery. Proc Natl Acad Sci. 2013;110(6):2082-7. In this work, non-invasive and efficient cytosolic delivery based on mechanical deformation was developed and tested with 11 cell types including embryonic stem cells and immune cells. This device showed a 10-fold improvement in colony formation comparing with previous transfection methods.

81. Xie X, Xu AM, Leal-Ortiz S, et al. Nanostraw electroporation system for highly efficient intracellular delivery and transfection. ACS Nano. 2013;7(5):4351-8.

82. Grigsby CL, Ho Y-P, Lin C, et al. Microfluidic preparation of polymer-nucleic acid nanocomplexes improves nonviral gene transfer. Sci Rep. 2013;3:3155.

83. Mali P, Esvelt KM, Church GM. Cas9 as a versatile tool for engineering biology. Nat Methods. 2013;10(10):957-63.

84. Zetsche B, Gootenberg JS, Abudayyeh OO, et al. Cpf1 is a single RNA-guided endonuclease of a class 2 CRISPR-Cas system. Cell; 2015. In Press Corrected Proof.

85. Li M, Suzuki K, Kim NY, et al. A cut above the rest: targeted genome editing technologies in human pluripotent stem cells. J Biol Chem. 2014;289(8):4594-9.
86. Hsu PD, Scott DA, Weinstein JA, et al. DNA targeting specificity of RNA-guided Cas9 nucleases. Nat Biotechnol. 2013;31(9):82732.

87.• Hou Z, Zhang Y, Propson NE, et al. Efficient genome engineering in human pluripotent stem cells using Cas9 from Neisseria meningitidis. Proc Natl Acad Sci. 2013;110(39):15644-9. In this paper, the Neisseria meningitidis Cas9 (NmCas9) system as a genome-editing platform was developed. The result showed $\sim 60 \%$ efficiency for human embryonic stem cell lines and expanded genomic contexts in eukaryotes for editing.

88. Davis RP, Nemes C, Varga E, et al. Generation of induced pluripotent stem cells from human foetal fibroblasts using the Sleeping Beauty transposon gene delivery system. Differentiation. 2013;86(1-2):30-7.

89. Kim H, Kim J-S. A guide to genome engineering with programmable nucleases. Nat Rev Genet. 2014;15(5):321-34.

90. Liu J, Gaj T, Wallen MC, et al. Improved cell-penetrating zincfinger nuclease proteins for precision genome engineering. Mol Ther Nucleic Acids. 2015;4, e232.

91. Jinek M, East A, Cheng A, et al. RNA-programmed genome editing in human cells. Elife. 2013;2, e00471.

92. Gaj T, Gersbach CA, Barbas III CF. ZFN, TALEN, and CRISPR/ Cas-based methods for genome engineering. Trends Biotechnol. 2013;31(7):397-405.

93. Choi S, Yu X, Jongpaiboonkit L, et al. Inorganic coatings for optimized non-viral transfection of stem cells. Sci Rep. 2013;3: 1567.

94. Hujaya SD, Marchioli G, Roelofs K, et al. Poly(amido amine)based multilayered thin films on 2D and 3D supports for surfacemediated cell transfection. J Control Release. 2015;205:181-9.

95. Li B, Yang J, Ma L, et al. Fabrication of poly(lactide-co-glycolide) scaffold filled with fibrin gel, mesenchymal stem cells, and poly(ethylene oxide)-b-poly(L-lysine)/TGF-beta1 plasmid DNA complexes for cartilage restoration in vivo. J Biomed Mater Res A. 2013;101(11):3097-108.

96. Low WC, Rujitanaroj PO, Lee DK, et al. Nanofibrous scaffoldmediated REST knockdown to enhance neuronal differentiation of stem cells. Biomaterials. 2013;34(14):3581-90.

97. Lu H, Lv L, Dai Y, et al. Porous chitosan scaffolds with embedded hyaluronic acid/chitosan/plasmid-DNA nanoparticles encoding TGF-beta 1 induce DNA controlled release, transfected chondrocytes, and promoted cell proliferation. PLoS One. 2013;8(7), e69950.

98. Monteiro N, Ribeiro D, Martins A, et al. Instructive nanofibrous scaffold comprising runt-related transcription factor 2 gene delivery for bone tissue engineering. ACS Nano. 2014;8(8):8082-94.

99. Tierney EG, Duffy GP, Hibbitts AJ, et al. The development of non-viral gene-activated matrices for bone regeneration using polyethyleneimine (PEI) and collagen-based scaffolds. J Control Release. 2012;158(2):304-11.

100. Yu X, Murphy WL. 3-D scaffold platform for optimized non-viral transfection of multipotent stem cells. J Mater Chem B Mater Biol Med. 2014;2(46):8186-93. 